\title{
E-Learning Certificate Using Digital Watermarking Technology
}

\author{
Ajlan S. Al-Ajlan \\ Information System Management, Qassim University, Buraydah 52571,Saudi Arabia
}

\begin{abstract}
There has been an increasing demand for e-learning technologies and especially for digital watermarking. Using the digital watermarking technology in e-learning is increasingly becoming an important part of the strategy for trusting on-line materials and activities, especially the on-line certificate both easy and cheaply in flexible e-learning way. This paper sets out to develop and create a novel approach to digital watermarking for e-learning certificate that can increase the trustworthiness of e-learning systems in institutions all over the world. This approach will enable these institutions to create an on-line certificate and to add a watermark that can be trusted by other institutions. The most important driving force is concern over protecting copyright, such as for video, audio and other works now available in digital form; it might be that the ease with which perfect copies can be made will lead to large-scale illegal copying, which will in turn undermine the creation of new books, films, pictures etc. the main aims of this paper is to introduce high-quality protected on-line certificates using two kinds of digital watermarking (visible digital watermarking and invisible digital watermarking) in e-learning systems. In addition, this study aims to enable institutions to offer e-learning certificate and to embed digital watermarking systematically.
\end{abstract}

Keywords: E-learning, Visible and Invisible Digital Watermarking, Moodle, E-Learning Certificate.

\section{Introduction}

E-learning is an interactive learning in which the learning content is available on-line and provides automatic feedback to the student's learning activities, and therefore using the Internet to enhance e-learning has become a trend in modern higher education institutes. For many years being able to hide digital information has captured the imagination of researchers, and therefore there has been an increasing demand for security technologies and especially for Digital Watermarking (DW). Using DW in e-learning is increasingly becoming an important part of the strategy for trusting an on-line e-learning certificate. It is a new technology, which could solve the problem of enforcing the copyright of content transmitted across shared networks. DW enables a copyright holder to put in a hidden message within images, pictures, sound files, and even raw text.

This paper sets out to develop and create a novel approach to DW for an on-line certificate that can increase the trustworthiness of e-learning systems in institutions all over the world. This approach will enable these institutions to create an on-line certificate and to add a watermark that can be trusted by other institutions. The most important driving force is concern over protecting copyright, such as for video, audio and other works now available in digital form; it might be that the ease with which perfect copies can be made will lead to largescale illegal copying, which will in turn undermine the creation of new books, films, pictures etc.

Since the beginning of the Internet, a number of individuals have presented their intellectual property on-line. They started off with just simple watermarking on photography, but then moved on to fields such as image, audio and video. DW technology is now widely applied to protect the ownership of digital images, audio files, videos, and even raw text. Thus, DW technology is used to address digital rights management, protect information, and conceal secrets. For e-learning that involves high-quality images, pdf files etc, DW technology can also enhance learning efficiency. Additionally, information hiding techniques provide an interesting challenge for digital forensic investigations.

The main objective of this study is to improve the use of DW for on-line certificates in e-learning. This approach will increase trust between institutions and allow different institutions in different countries to work together and share on-line certificates. The main aims of this paper is here listed below: (1) develop and create high-quality protected on-line certificates using an efficient DW that can increase trustworthiness in e-learning all over the world, (2) to enable e-learning systems to create an on-line DW and embedded watermarking system so that responsible users (teachers or administrators) can certificate documents automatically, (3) some of e-learning systems do not have full certificates for all courses, such as Moodle, which has just a transcript for each course with its details. This study provides a full certificate for all courses for every student. In addition, the responsible person can add watermarking automatically, (4) this approach will open a wide door for collaborative e-learning on-line certificates, which will increase trust between institutions and will enable them to use the Internet to send their certificates as originals, (5) to find the most suitable technology for supporting the worldwide trend of offering on-line certificates over the Internet, which includes institutes in a wide variety of countries, in order that they can exchange on-line certificates both easily and cheaply in a flexible manner. 
This paper is structured as follows: in the beginning, we present the related studies including a literature review of DW and e-learning, as well as, Moodle as the area of study in Section 2 and Section 3. Section 4 highlights on architecture of e-learning certificate. An implementation of e-learning certificate using digital watermarking is presented on Section 5. An analysis and evaluation of e-learning certificate system (ECS) is presented in Section 6. Finally, the conclusion and future work are in Section 7.

\section{Digital Watermark}

The digital information revolution has altered commercial and academic behaviour, and the Internet has played an important role in this revolution all over the world. The benefits through the broadcasting of data, easy editing of any kind of digital content, and the ability to reproduce digital content without any loss in quality, have made digital technology far superior to the old analogue systems $[1,2,3]$. This has generated new challenges and new opportunities for innovation. The development and growth of novel IT technologies for the rapid provision of services to both academia and industry over the Internet has resulted in a demand for dependable and well-protected copyright protection techniques for many kinds of data. For the prevention of unauthorized tampering of Digital Multimedia Data (DMD), DW is being developed to enhance the protection and copyright of this data. In recent years, DW has shown significant progress $[3,4,5]$.

DW is a powerful technology for identifying copyright data. It is digitized information such as text, images, audio, and video that is embedded in the original information, and can be extracted correctly by a watermark extracting method to prove copyright ownership. Moreover, it is not easy for the human eye to notice the difference between original information and one with a watermark [5]. DW enables an individual to add hidden copyright messages to digital audio, video, images and text. It is a method for marking data sets and embedding a mark for ownership registration based on the human visual system. DW is a considerable challenge for the world's largest computer companies, such as International Business Machines and Intel. DW has two major research topic areas, which are: 1) the Visible Digital Watermark (VDW) technique, and 2) the Invisible Digital Watermark (IDW) technique. The VDW technique is not popular because it affects the quality of an image. On the other hand, IDW has made considerable progress in recent years and has been received favourably by both academia and industry $[6.7,8,9]$.

The most important aspects of embedded IDW can be divided into three characteristic features, and they are [10]: (1) Unobtrusiveness: the human eye cannot see the watermark, and it does not affect the quality of the original image, audio, etc, (2) Robustness: the embedded watermark is still present in the image even if that image is compressed or processed and (3) unambiguous: Revealing the watermark unmistakably identities the original image, audio, etc. DW embeds information as machine readable within the content of digital media such as video, audio and images the data is encoded through slight changes to the format of the media file. Typically, these alterations will not be perceptible to normal users, and neither will the DW within the content, but they may be to designers.

DW can embed in: (1) higher frequency, (2) middle frequency and (3) lower frequency. The problem in higher frequency is that DW does not decompress back to $100 \%$ of its original because the high level of compression involved produces very small compressed files; there is some data quantity loss when decompressed files are restored, and this also affects DW. The increasing demand for Digital Multimedia Technology (DMT) and wireless applications is very apparent on the Internet, because currently, the distribution of DMT data is much faster and easier though the Internet. Accordingly, DW, because of its uniqueness, has become an important issue facing developers when they send and receive data over the Internet.

DW technology offers many new advantages compared to its old analogue counterpart. In this section, we assess the advantages and disadvantages of DW, and we focus on its advantages for the field of e-learning. This section starts with the advantages of DW, as below [4, 10, 11]: (1) DW enables institutions to trust each other by using an on-line certificate that has watermarking. This is achieved in this paper, and so institutions can insert and embed VDW and IDW into their certificates, (2) The most important driving force is concern over the protection of copyright for such works as digital images, video, audio and even raw text, that are available in digital form. It is now easy to make perfect digital copies, and large-scale illegal copying undermines all industries involved in books, films, and even academic publications, (3) for e-learning, high-quality images etc, DW technology can enhance learning efficiency. DW technology can be used in academia to address digital rights management, protect information, and conceal secrets. Information hiding techniques provide an interesting challenge for digital forensic investigations and (4) DW enables institutions in different countries to work together and share and use certificates without fear because DW reduces the forgery and fraud in certification all over the world.

On the other hand, DW has some disadvantages and we mention some of them, as follows: (1) attacking DW is simple; it can be corrupted or eliminated if the embedded watermark is in a lower frequency, (2) with DW, the quality of the image can be affected and (3) With DW, especially IDW, the size of the document is increased. 


\subsection{Architecture Of Digital Watermark}

DW is aimed to complement cryptographic procedures, and it is possible for a DW to be a visible or invisible identification code, which is permanently embedded in DMD, and which remains present within the data following any decryption procedure [11]. The concept of DW comes from the slightly visible watermark of a company's name that can be imprinted into paper for copyright or identification purposes. Accordingly, the important benefit of DW is to copyright information and to identify the file. DW is the process of embedding a bits pattern in a digital text video, image or audio but, in this case, the bits pattern will be hidden and difficult to alter. IDW enhances a copyright defence, therefore guarding and guaranteeing the digital work [12].

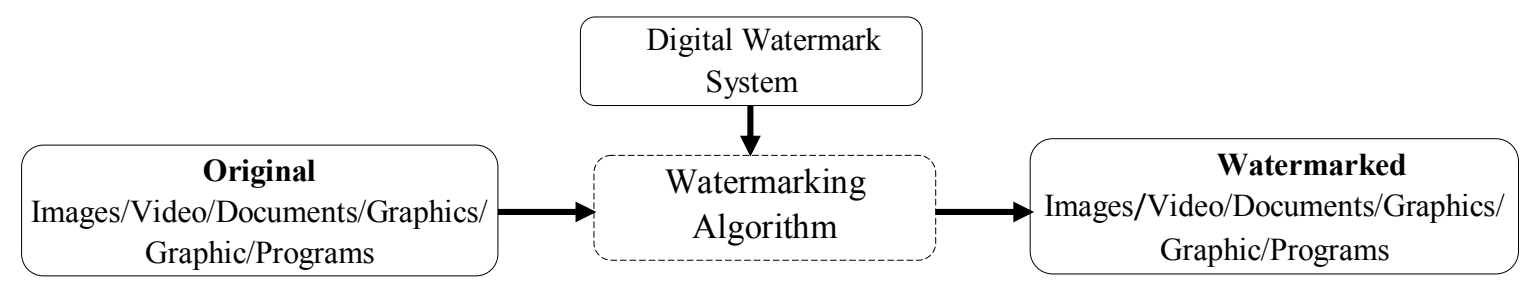

Figure 1: Digital Watermark System

DW is the use of perceptually visible and invisible authentication techniques but which control the distortion that is introduced into DMD. The scenario of DW is described in Fig. 1 where an owner places original elements (images, video, documents, graphics, or programs) on a network server and wants to "protect" them. Multimedia Security Applications (MSA) is used to enhance the protection and copyright of DMD. In recent years, MSA has shown significant progress and it includes: (1) Copyright Protection, (2) Privacy, (3) Intruder Detection, (4) Forgery Detection, (5) Proof of Delivery, and (6) Proof of Purchase. In addition, MSA has tools and they are: (1) Authentication, (2) Encryption, (3) Time-stamping, (4) Hashing, and (5) DW. Fig. 2 is a diagram from Canon $(\mathbb{C}$ website that gives a simplified explanation of DW and its scenario [12].

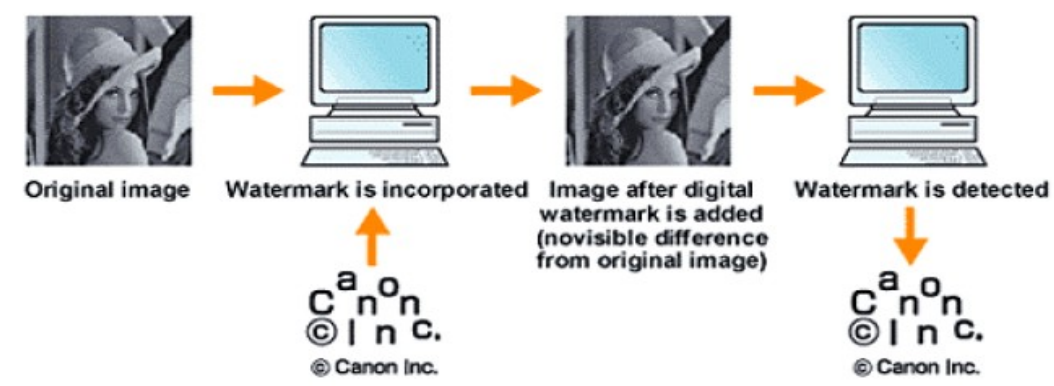

Figure 2: A simplified explanation of digital watermarking [12]

Currently, the most important tool available to protect data is encryption. This tool defends data throughout communication between sender and receiver over the Internet. After receiving data and decoding (decryption), the data is no longer protected. Thus, DW is complementary to encryption. DW has two main phases [10]: (1) the embedding of DW and (2) the extraction of DW.

\subsection{Types of Digital Watermarking}

DW can be an institutional logo or a pseudo random sequence or background of an image and pfd file. The data that has DW may be compressed to decrease the size but it may be corrupted by noise throughout its broadcast through a noisy channel. We can classify DWs into two categories: (1) Visible Digital watermarking (VDW) and (2) Invisible Digital watermarking (IDW) [13].

Most studies have researched invisible digital watermarking (IDW) as it is used more in DMD these days. Visible digital watermark (VDW) has been traced back to the end of 13 th century $[4,9]$. In this section, we will concentrate on IDW and VDW as this paper tests both types with e-learning certificate [13].

Visible Digital Watermark: VDW was the initial and mainly primitive method of watermarking, and covers the object that has a watermark, making it visible on this object for users. While VDW has been used for a long time, it is not a protected form of watermark for DMD. The algorithm technique is employed for VDW but this is not sufficient for protecting the DMD. This kind of watermark can only be utilized for holder identification purposes. In contrast, IDW is used in all other DMD.

VDW is able to be seen as transparent in DMD, which is covered on the primary DMD. Including the logo and seal of an institution, which holds the rights to the primary DMD, it enables the primary DMD to be 
seen; it marks it obviously as the property of the institution. It is important that the removing the VDW is difficult; however, it is easy for the owners.

Invisible Digital Watermark: IDW can be executed via various techniques and the easiest part is hiding a watermark within DMD. The benefits of hiding are that even if a piece of the image is cropped, the recipient can still obtain the needed message as the watermark is embedded into message many times. In this case, the message is small, in contrast to the original message [4]. For example, each pixel is represented by 8 bits (10001100), and the most significant bits are to the left and the least significant bits are to the right. If we change the most significant bits, it will have a big impact on the colour. In contrast, if we change the least significant bits, it will have a minimal effect. To be clear, if we change only one or two least significant bits, it will have a minimal effect because the human eye can only notice about six bits of colour the human eye cannot note the difference in the last two bits being changed. For example, if we deal with 10001100 and alter it to 10001111 , it will be the same colour to the human eye. So we only embed data in those bits [4].

When a DMD that has watermarking is destroyed by manipulating it digitally, an IDW may still be useful in proving the authenticity of this DMD. If the DW remains unbroken, then the DMD is not lost, but if a DW is destroyed, then the DMD is indeed lost. IDW is resistant to damage under any DMD manipulation, making it useful in confirming the ownership of the DMD suspected of fraud [14].

\section{E-learning Systems}

E-learning is a term used to demote computer enhanced learning, and it is used interchangeably in many contexts, but it is critical to be clear what one means when one talks of e-learning [15, 20]. E-learning systems are a group effort, where administrators, educators, students, guests and all other users from divers areas of expertise come together in order to serve a community of learners $[17,18]$. In order for present and future generations of personalized e-learning to progress and improve learning effectiveness and efficiency, some vital requirements must be understood. In the last decade, more than 300 e-learning systems, of which more than 60 are Open Source Software (OSS), have been developed by universities [19, 20, 21, 22].

The most important reason why e-learning has become popular in many institutions all over the world is that because there are actual benefits to be gained from the use of e-learning technology and they are [17, 23]: (1) teacher can use e-learning to manage their courses including timetable, announcements, tests, tracking student progress, assignments, and so on, (2) it allows user to upload and download files onto the e-learning system for everyone and anytime, (3) it enables interaction among teachers and students totally free of time and location, (4) it enables easy on-line delivery of materials, which are easy to use for both lecturers and students and (5) student can enjoy the privacy at home, from anywhere, currently, the Internet offers cheap and easy access to information.

\subsection{E-learning Architecture}

Currently, there is no consistent standard architecture for e-learning products. Most architectures depend on the type of e-learning system and the institution's requirements.

An e-learning environment works as a portal for VLE tools including support, technical specifications and learner tools as shown in Fig. 3. The diagram above is based on the widely circulated one created by BECTa in 1999. The most important target for this diagram is to position VLE as a sub-system within the e-learning environment [15].

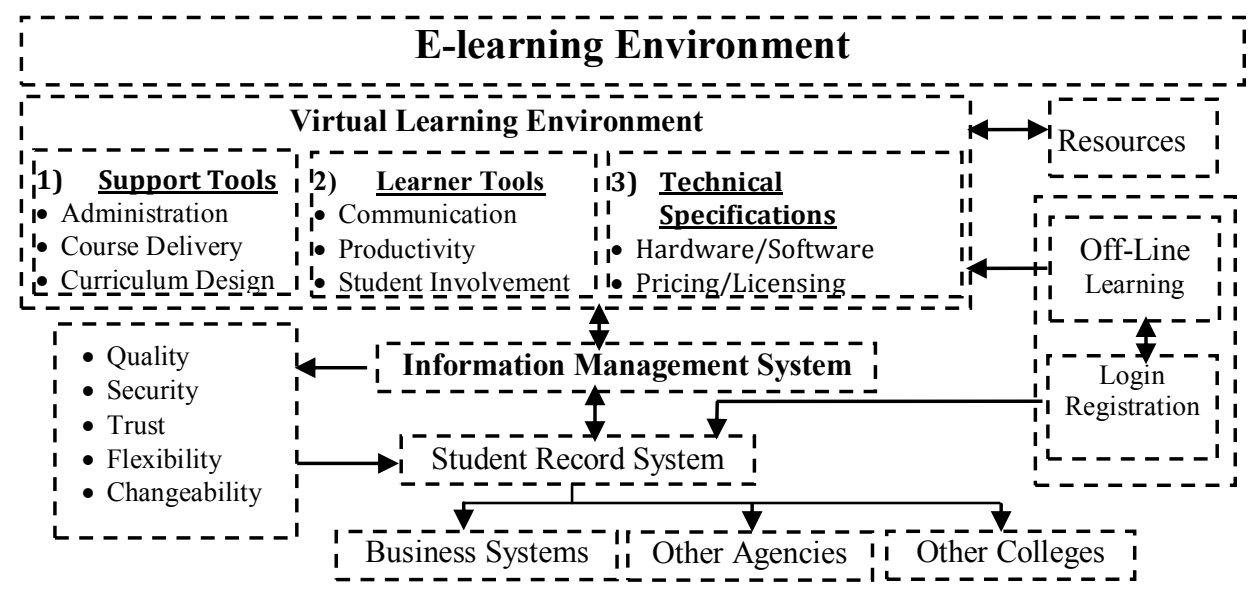

Figure 3: The E-learning Environment [24] 


\subsection{Moodle As A Case Study}

Moodle is one of the most user-friendly free open-source products available all over the world. It is an e-learning system that allows user to provide and share assignments, forums, documents, chat, quizzes, etc. with learners in an easy educational process. It is a Content Management System (CMS) designed to assist teachers in creating quality on-line courses. [21]. Now, Moodle has a huge user community with over $64,368,175$ registered users on this site alone, speaking 78 languages, and currently 94,941 Moodle sites from around 235 countries have been registered [21]. Martin Dougiamas created Moodle while he was working on a postgraduate degree at the Curtin University of Technology in Australia. [25].

This section mentions the reasons for choosing Moodle as the area of study and analysis. The most important reasons for choosing this package are [21, 16, 26]: (1) Moodle is Open Source Software (OSS) and this means users are free to use it, download it, modify it and even distribute it under the GNU license, (2) it is a CMS and a Virtual Learning Environment (VLE) that allows tutors to provide and share assignments, documents, forums, quizzes, etc. with their students, (3) it can be used on almost all servers that can use PHP. Moodle runs without modification on Windows, Unix, and any other system that supports PHP. It has excellent documentation, and strong support for security and administration; it is also easy to upgrade from one version to the next. Data is stored in a single database; PostgreSQL or MySQL are best but it also supports Access, Oracle, (4) The most important feature of Moodle is that has been developed with both pedagogy and technology.

The Moodle architecture sets a brilliant precedent for good practice, with low coupling and high consistency, which the other LMSs have not succeeded in achieving. This gives system simplicity and flexibility, making it easier to be further developed [21]. The Moodle architecture is described in Fig. 4 including the Moodle site and its components. This study has added a new layer that provides a generic solution for migrating any stand-alone into a DW certificate of e-learning. This layer has three main functions: (1) create certificate, (2) insert VDW, and (3) embed IDW as in the left top in Fig. 4.

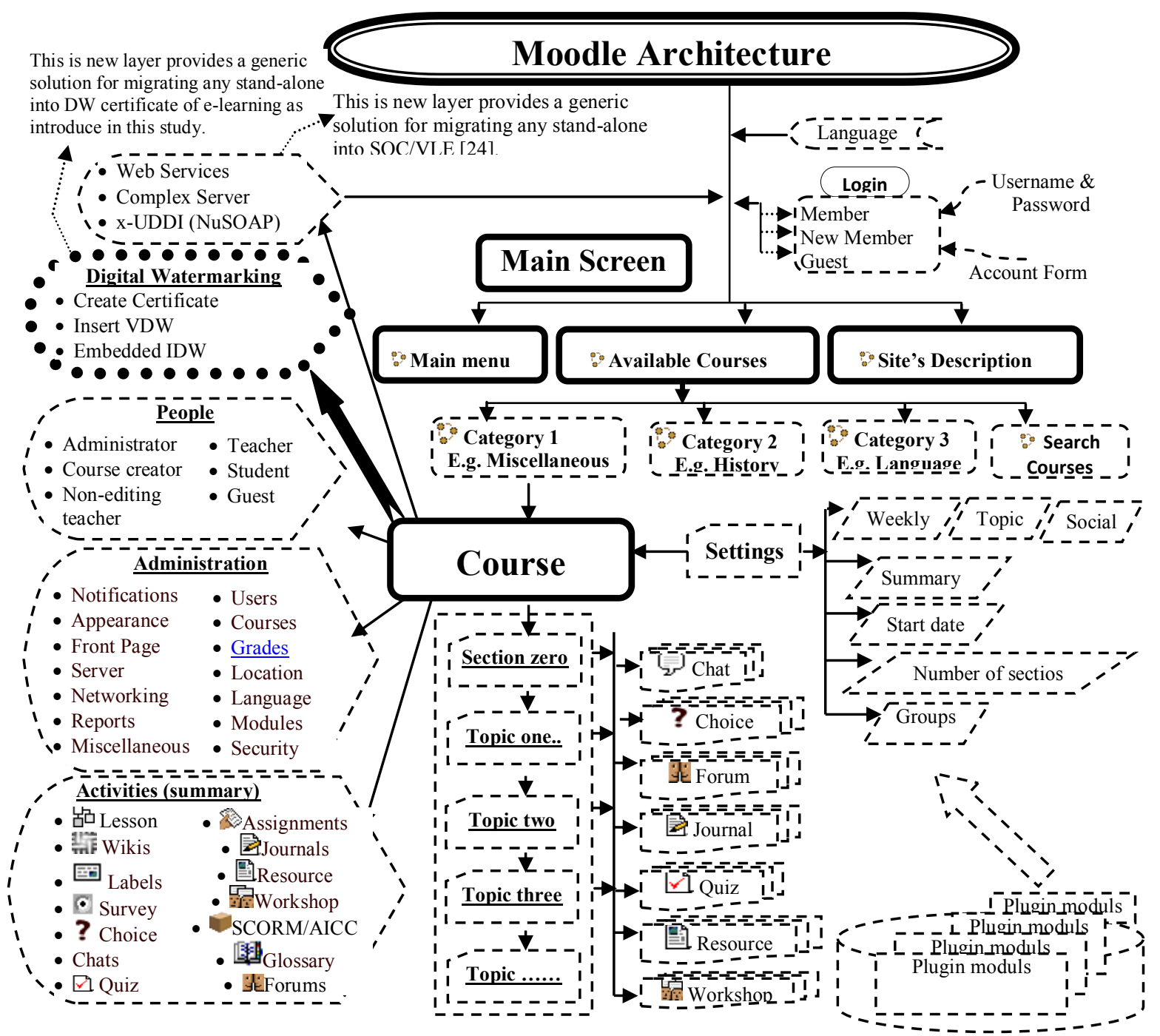

Figure 4: Architecture of Moodle. 


\section{Architecture of E-learning Certificate}

Defining a model makes it easier to break up a complex application or software into simple pieces that can be individually studied. A model lets developers and designers more easily spotlight the smaller parts of a system. However, the need for a strong methodological base for the development of DW has raised the need for modelling and method techniques that can promise quality in the development of these kinds of applications and software, and in this regard the importance of the Unified Modelling Language (UML) for process modelling in DW schemes has been underplayed [18].

Before determining the architecture of e-learning certificates based on DW, we should decide what technical requirements are needed to design our approach. Technical requirements are technical aspects that need to be satisfied before something is deemed possible. At this point, the technical requirements of the architecture of e-learning certificates based on DW are of two categories: functional and non-functional requirements. Functional requirements are a piece of the answer to a question around why some process has happened in the software, and they evolve through a process of selecting goals [27]. In contrast, non-functional requirements state criteria that can be used to referee the operation of a software rather than specific behaviours.

\subsection{Architecture of Digital Watermark}

DW is aimed to complement cryptographic procedures and it is possible to use a visible or invisible identification code, which is permanently embedded in the Digital Multimedia Data (DMD) and remains present within the data following any decryption procedure [11].

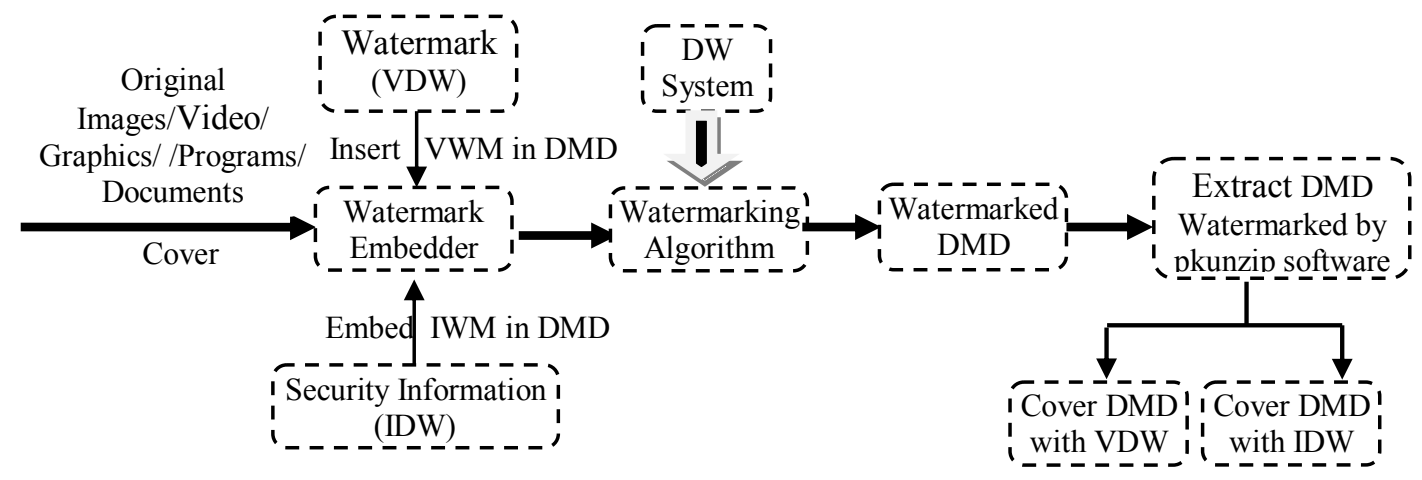

Figure 5: The Typical DW System.

The idea of DW is that it should result in a visible watermark, but principally it is a technique that is used to convey data that is embedded within the cover DMD. A popular reason for using DW is to give proof of ownership. It is clear that DW should be robust against any efforts that may try to manipulate or eliminate it [11]. Fig. 5 displays a typical DW system, which includes a watermark embedder and an extractor. The inputs to the watermark embedder are watermark (VDW), the cover DMD and security information (IDW). The watermark can be background, image, a number sequence or a binary bit sequence. The security information is used to improve the security of DW. The output of DW system is the watermarked data as in Fig. 10.

Visible Digital Watermark: VDW was the initial and mainly primitive method of watermarking, and covers the object that has a watermark, making it visible on this object for users. While VDW has been used for a long time, it is not a protected form of watermark for DMD. VDW is able to be seen as transparent in DMD, which is covered on the primary DMD. Including the logo and seal of an institution, which holds the rights to the primary DMD, it enables the primary DMD to be seen; it marks it obviously as the property of the institution. It is important that removing the VDW is difficult; however, it is easy for the owners.

As in Fig. 5, inserting a VDW into the DMD into the on-line certificate is the first target of our system. Users will be able insert a VDW, which could be logo, stamp, or text into the cover DMD, which in our example is a certificate. Fig. 10 shows that the certificate has the text "De Montfort University" and the stamp of De Montfort University. Thus, when users see the VDW on the on-line certificate, they will be sure that this certificate has been issued by De Montfort University.

Invisible Digital Watermark: IDW can be executed via various techniques and the easiest part is hiding a watermark within DMD. The benefits of hiding are that even if a piece of the image is cropped, the recipient can still obtain the needed message as the watermark is embedded into the message many times. IDW is a cover DMD that cannot be seen by the human eye, but can be discovered by an algorithm [14].

Fig. 1 shows the embedding of IDM in DMD, which is included with the on-line certificate, and is the second target of our system. User will be able to hide information within the certificate file, and this can be file 
(text or word) or image. Normal users will not be able to see this information or extract it from the certificate, but they will be able to see the VDW, and this will deceive them into thinking that this certificate has only a VDW. In addition, this kind of watermarking has a private key, which makes the certificate more secure and increases the trustworthiness among institutions.

Extracting a Watermark from DMD by pkunzip Software: After embedding information into a certificate, we need to extract this information. Therefore, we create a new program that can extract information that has been embedded into a certificate or into any DMD format and this software is pkunzip.

\subsection{Requirements for E-learning Certificates}

This section emphasizes the functional and non-functional requirements of e-learning certificates (to be used in Moodle) based on DW. Certain features are taken from the certificate tools that we use, and are integrated into Moodle activities; these allow for the integration and upload of on-line certificates. This approach also allows for the automatic marking of certificates with the stamp of any institution that uses Moodle. The functional and non-functional requirements of e-learning certificates (Moodle) based on DW are:

\subsubsection{Functional Requirements}

The definition of a functional requirement is that the principle design of a paper is based on the aims of that paper. It explains why some particular process or object occurs in a system, which has been developed through a process of specifying exact targets. This kind of requirement shows functionality in precise bulleted items that accurately identify what a certain service achieves. Service methods, operations, actions etc, have to be defined in terms of what piece of the functionality it offers [27].

As in Fig. 5, this paper involves marking on-line certificates by using DW technology. The most important driving force is concern over protecting copyright, such as for video, audio and other works now available in digital form; it might be that the ease with which perfect copies can be made will lead to large-scale illegal copying, which will in turn undermine the creation of new books, films, pictures etc. DW in on-line certificates has some particular functional requirements that enable institutions to create an on-line certificate and to add a watermark that can be trusted by other institutions, and these functional requirements are as below: On-line Certificate: recently, e-learning systems have forced institutions to manage on-line certification within their own differing cultures. Therefore, this paper presents a simple certificate that can be used in all e-learning systems, and which displays all courses. We offer this because some e-learning systems do not have certificates for all courses, and just have a transcript for each course with its details. As in Fig. 9, the on-line certificate has two columns. One of them is for the course and the other for the grade. We kept this certificate simple so as to enable us to apply it to other e-learning systems and to insert DWs easily. It is simple to update and modify this certificate and to add more columns and rows or to delete them.

FPDF Class: it is a PHP class that enables PDF files to be generated with pure PHP. It is free and users can modify and distribute it to suit their needs under the license: FPDF. It is possible to use it without using the PDFlib library. Another advantage of FPDF is that has high-level functions. Also, it requires no extension, except GD for GIF support and zlib to activate compression [28].

Class PDF_Rotate: This class enables performing a rotation about a given centre. The script to set a rotation is: Rotate( $\$$ angle, $\$ \mathrm{x}=-1, \$ \mathrm{y}=-1)$

Table 1: sample and description of PDF Rotate

\begin{tabular}{|c|c|c|}
\hline No & Sample & Description \\
\hline & Angle & Angle in degrees \\
\hline & $\mathrm{X}$ & Abscissa of the rotation centre. Default value: current position \\
\hline & $\mathrm{Y}$ & Ordinate of the rotation centre. Default value: current position \\
\hline
\end{tabular}

PDF Rotate involves all elements that are printed after the call method through the exception areas. The methods [GetX() and GetY()] are not involved, nor the automatic page break machine. PDF Rotate is not reserved from page to page and each page starts with a null rotation [28].

This study uses a PDF Rotate with an on-line certificate as a VDW and the script that we use to rotate VDW in an on-line certificate, which is "De Montfort University", as in Fig. 10.

Database Query: Moodle uses Structured Query Language (SQL), which enables clients to access data in relational database management systems, for example Oracle, Microsoft SQL Server, Informix, and Sybase. It permits clients to explain the data that the requestor wants to see, and it defines the data in that database. Moodle has more than 200 tables in Version 2.1.2, which this paper has used. Each database has select query and this depends on some of the Moodle tables and the relationships between them. For example, a certificate for a student with all courses has a long query and six tables, and the relationships between these tables are displayed in Fig. 8 [8]. 
Database is an important requirement as it is responsible for loading data from DB to create an on-line certificate. Currently, Moodle does not have on-line certification, therefore, we create a query, which is loading data from DB to the on-line certificate for all modules in Moodle.

\subsubsection{Non-Functional Requirements}

This kind of requirement identifies the criteria that can be used to judge the process of the system, rather than precise behaviours. Non-functional requirements usually contain flexibility, security, trust, changeability, extensibility, reliability, availability and quality of service issues. This part details the nonfunctional requirements that are required when on-line certificates use DW, and these requirements are:

Trust: Trust determines the specifications for secure mechanisms to facilitate trusting relationships in diverse DW environments. For a secure communication between two institutions, both must exchange security credentials using a specification. Trust is 'confident reliance' in that we may have confidence user, events, or at least in our beliefs, but if we do not rely on them, our confidence alone does not amount to trust [29].

Security: Security is a significant issue that every developer and researcher has to be concerned with when writing and designing a system. In the on-line certificate system, the security issue is most important because if this system not secure, no institution will trust this on-line certificate. Therefore, we have made every effort to build this system to a high level of security, and therefore we use VDW and IDW steganography [30].

Security specifies the level of on-line certificate confidentiality and non-repudiation by authenticating the parties involved, encrypting messages, and maintaining access control. An on-line certificate has different security levels and mechanisms depending on the needs of the institutions [30].

Flexibility: Flexibility refers to the availability of alternative resources and may have diverse parameters, especially those related to physical and operating systems. Flexibility is one of the most significant advantages of using DW with on-line certificates. The on-line certificate is accessible using PHP, which offers more flexibility to developers because they can use the technology that they are most comfortable with [24,31].

\section{E-learning Certificate Implementation}

The possibility of a user knowing the algorithm for detecting or decoding DW is negligible. In this paper, the idea is to embed security data, which is txt, with the certificate, which is pdf. Then, the certificate will be as a one file, which is a pdf file. The security txt file will be embedded inside the pdf and the user cannot see it. To open and extract the security txt file, we will give institutions a small program to enable them to extract the certificate and get the certificate pdf file and the security txt file separately. If anyone tries to open the certificate without our program, it will be damaged.

\subsection{E-learning Certificate System}

An on-line certificate has become more significant in modern e-learning systems. Many developers and researchers have expended a great deal of effort in designing e-learning products with on-line certificates. Therefore, this study has created an on-line certificate system for any e-learning product that does not have an on-line certificate. The most significant advantage of the on-line certificate is that it is applicable for any elearning product.

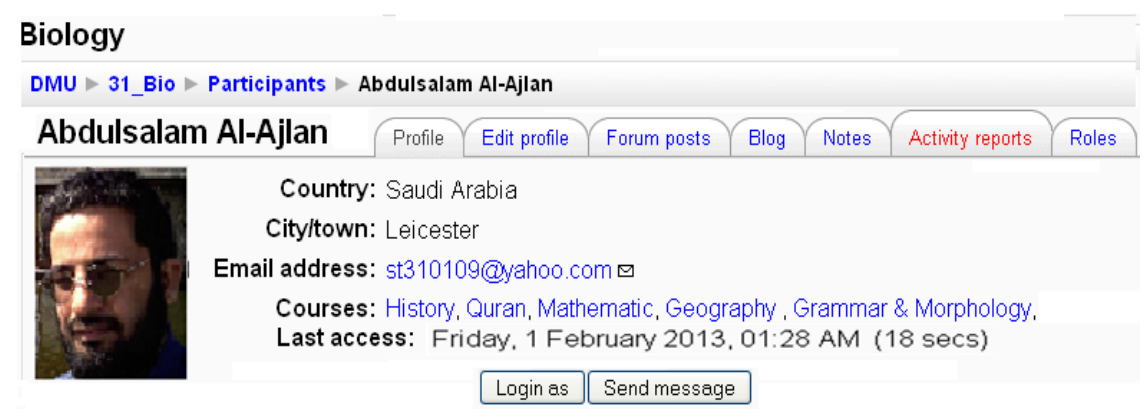

Figure 6: on-line Certificate under Activity report page.

As in Fig. 6, we create an on-line certificate for e-learning Moodle under the "Activity report" page; it is also in Account, so students and teachers can access this certificate by following either of two paths: (1) Course categories $=>$ Course $=>$ Participants $\Rightarrow$ The name of student $=>$ Activity report $=>$ Grade $=>$ View Certificate and (2) Users $=>$ Accounts $=>$ Browse list of users $\Rightarrow>$ The name of student $=>$ Activity report $=>$ Grade $=>$ View Certificate. 
As we can see in Fig. 7, Moodle just issues a certificate as a report for only one course, which is "Biology". On the other hand, this study has succeeded in issuing a certificate for all courses, and this is opened when the teacher presses on the "View Certificate" button, as in Fig. 10.

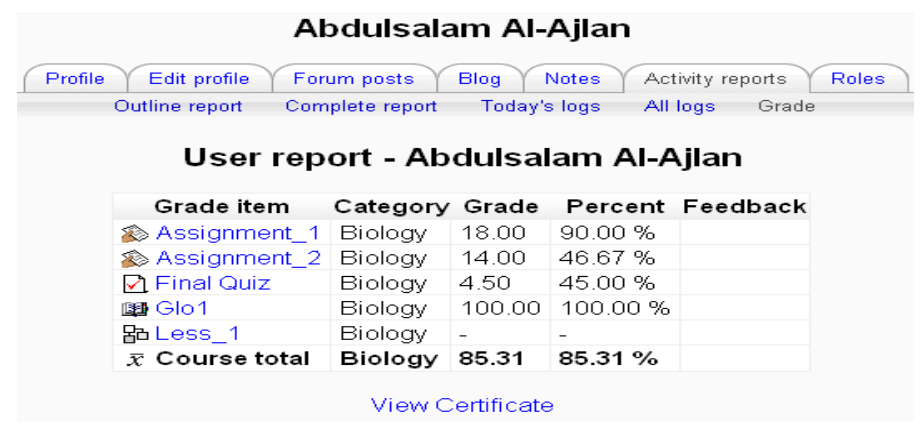

Figure 7: on-line Certificate for only one course

To view the certificate we have chosen a suitable location, which is under the "Grade" page, as in Fig 10. The certificate works when the teacher presses on the "View Certificate" button, as below in Fig. 7. The code responsible for commanding is created, and also we added a link to 'view certificate' and a link to the showpdf.php file in order to open the pdf.

\subsection{E-learning Certificate Implementation}

When the on-line certificate is ready, the second target of our paper is to insert and embed DW within it. The important advantages of DW are that it protects information, manages digital rights, and conceals secrets, and in the context of e-learning, that involves high-quality images, pdf files etc. At the moment, the on-line certificate is very simple, as in Fig. 10. This certificate is a student transcript and has two columns, which are course and grade. The most important benefit of ECS is that responsible users (usually teachers or administrators) can certify an on-line certificate automatically by pressing on the "View Certificate" button, as above in Fig. 7. After that ECS will perform two functions, which are: (1) create an on-line certificate and (2) insert VDW and embed IDW.

ECS has been added to the e-learning Moodle software as a watermark folder under the main folder of Moodl. The Watermark folder has many files and folders, and this section will mention them and give a brief description for each as follows:

Conectmysql.php File: Database is an important requirement as it is responsible for loading data from DB to create an on-line certificate. Currently, Moodle does not have an on-line certificate, therefore, we create query, which loads data from DB to the on-line certificate for all modules in Moodle. It uses Structured Query Language (SQL), which lets users access data in relational database management systems, for example Microsoft SQL Server, Oracle, Sybase, Informix, and others. It allows users to describe the data that the requestor wants to see, and it defines and manipulates the data in that database [21].

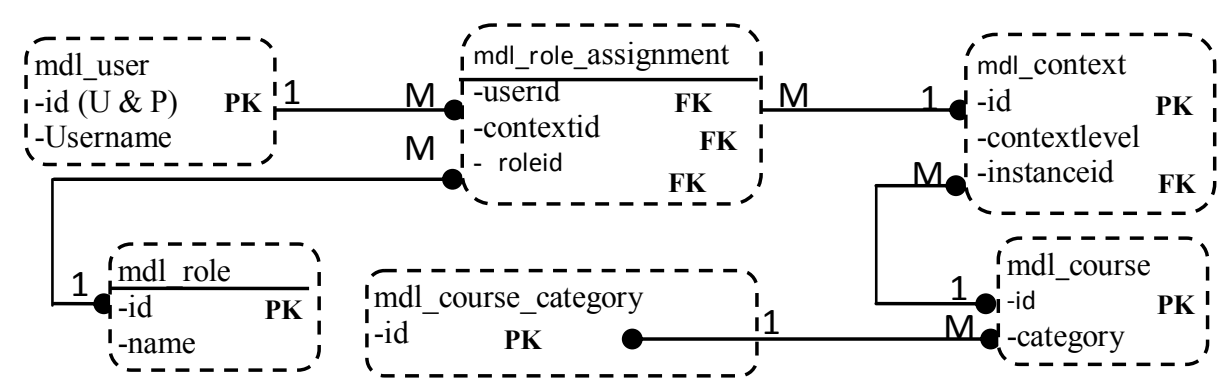

Figure 8: The Relationship of the Select Query of on-line Certificate in Moodle.

Moodle has a very large database (more than 212 tables in Version 2.4.2), which this paper has used. Each database service has select query and this select depends on some of the Moodle tables and the relationships between them. For example, a certificate for a student with all courses has a long query and six tables, and the relationships between these tables are displayed in Fig. 8.

Fpdf.CSS File: Cascading Style Sheets (CSS) define how to display HTML elements. Styles were added to HTML 4.0 to solve a problem. External Style Sheets can save a lot of work. External Style Sheets are stored in CSS files. We use this language to enable us to change the appearance and layout of our system, just by editing one single file. 
FPDF.php File: FPDF.php file version 1.6 was created by Olivier Plathey on 2008-08-03 and it has one class, which is a PHP class that enables PDF files to be generated with pure PHP. This file is free and users can modify and distribute it to suit their needs under the license: FPDF. It is possible to use it without using the PDFlib library. Another advantage of FPDF is that it has high-level functions. Also, it requires no extension, except GD for GIF support and zlib for activate compression, and it works with PHP 5 and PHP 4. The latest version of FPDF requires at least PHP 4.3.1. This class has two kinds of methods and they are [28]: (1) Public methods (has 47 functions) and (2) Protected methods (has 29 functions)

Rotation.php File: This class enables performing a rotation about a given centre. PDF Rotate involves all elements that are printed after the call method through the exception areas. The methods [GetX() and GetY()] are not involved, nor the automatic page break machine. PDF Rotate is not reserved from page to page and each page starts with a null rotation. We use a PDF Rotate with an e-learning certificate as a VDW in an e-learning certificate, which is "De Montfort University", as in Fig. 10 [28].

Showpdf.php File: This file is the heart of ECS and it is the centre of our paper, because it has one class that has the most important function in our work. In addition, it has a connect database command and sends our queries to the Moodle database. This file has one class and contains many functions as follows:

user.php File: This file is a user file and has all information related to users, such as activity reports for a course. We modified this file to be applicable with our paper and we added a link to view certificate and a link to showpdf.php file in order to open the pdf.

attach_detach Folder: This file has our programs 'attach' and 'detach' to embed IDW to an e-learning certificate. In addition, it has the pkunzip software that helps our programs attach and detach to perform correctly. Moreover, it has the original certificate pdf file, after being created by our ECS technique. Finally, it has the security code, after our programs (attach and detach) have created it.

\section{Analysis and Evaluation}

The architecture of software is normally complex, and the analysis and evaluation of software are most significant procedures for breaking any difficult part into smaller parts in order to gain a better understanding of the software. The purpose of analysis and evaluation in on-line certificates with DW software is to gain an improved understanding of the requirements of the issuing institutions in order that the on-line certificate software is sufficiently able to meet those requirements. The most important purpose of this paper is to examine ECS analysis and evaluation models. It will focus on the value of this research study to the research society, teachers and students.

\subsection{The Research Study Value}

This section will focus on the research value for the research society, educators and students. the final result of the questionnaires have confirmed that the ECS satisfies all three kinds of user, especially the teachers and students as in table 2 . most of the users agree and are very happy with this system.

Table 2: The Final Result for all Questions for all Users

\begin{tabular}{|c|c|c|c|c|c|c|}
\hline Questionnaires & User & Strongly Agree & Agree & Normal & Disagree & Strongly Disagree \\
\hline \multirow{3}{*}{$\begin{array}{c}\text { What is your } \\
\text { impression of the site? }\end{array}$} & The Research Society & 9 & 21 & 16 & 4 & 0 \\
\hline & teachers & 9 & 23 & 32 & 5 & 1 \\
\hline & Student & 22 & 32 & 38 & 5 & 3 \\
\hline \multirow{3}{*}{$\begin{array}{l}\text { Are you satisfied with } \\
\text { the interface of the } \\
\text { result? }\end{array}$} & The Research Society & 13 & 15 & 11 & 7 & 4 \\
\hline & teachers & 11 & 30 & 22 & 4 & 3 \\
\hline & Student & 19 & 27 & 38 & 10 & 6 \\
\hline \multirow{3}{*}{$\begin{array}{c}\text { Is this system easy to } \\
\text { use? }\end{array}$} & The Research Society & 10 & 11 & 23 & 5 & 1 \\
\hline & teachers & 18 & 20 & 26 & 4 & 2 \\
\hline & Student & 25 & 29 & 34 & 8 & 4 \\
\hline \multirow[t]{3}{*}{ Is this system secure? } & The Research Society & 6 & 18 & 26 & 0 & 0 \\
\hline & teachers & 11 & 20 & 36 & 3 & 0 \\
\hline & Student & 14 & 24 & 53 & 7 & 2 \\
\hline \multirow{3}{*}{$\begin{array}{l}\text { Does this system meet } \\
\text { overall goals? }\end{array}$} & The Research Society & 10 & 16 & 17 & 4 & 3 \\
\hline & teachers & 9 & 35 & 18 & 5 & 3 \\
\hline & Student & 27 & 37 & 33 & 2 & 1 \\
\hline \multicolumn{2}{|c|}{ Total } & 213 & 358 & 423 & 73 & 33 \\
\hline
\end{tabular}

Table 3 summarises all users' answers in this questionnaire. It proves that the most of users are happy as in columns "Agree" and "Normal". Table 3 displays the final questionnaire results for all users. There are 213 users who strongly agree with the ECS, and in contrast, there are just 33 who strongly disagree. In addition, there are 358 who agree with the ECS, but only 73 who disagree. Finally, there are 423 who said that the ECS is normal. 


\subsection{Analysis of E-learning Certificate System}

The architecture of the ECS, as in Fig. 5 above, lays the conceptual foundation for establishing interoperable DW in e-learning. This section will analyse the most significant part in our ECS in order to measure the quality of this system. The analysis will examine the system and explain the function of each part and the relationships between them.

An on-line certificate has been created by us, during the research on this study, for any e-learning system that does not have an on-line certificate. The most important benefit of this certificate is that it is applicable to any e-learning software. The target of on-line certificate is to give students a certified outcome for their study. When students graduate, the institution issues a certificate that confirms that these students have completed the course successfully, with marks for all modules that they have taken during the course.

At the moment, the ECS has a very simple certificate, as in Fig. 9 below. This certificate is a student transcript and has two columns, which are course and grade. ECS has the flexibility to extend the on-line certificate for more than just courses, and can add more columns. In addition, it has the ability to change the appearance and themes of the certificate and to meet the particular needs of each institution.

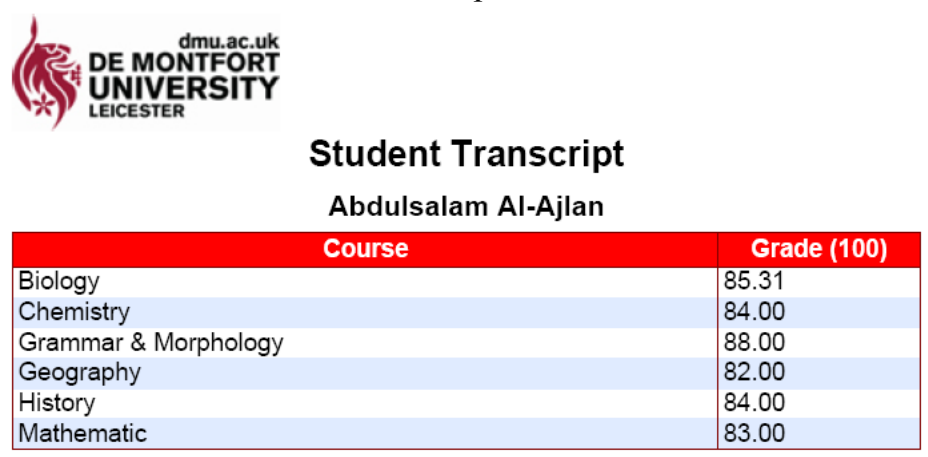

Figure 9: on-line Certificate.

Digital Watermarking with on-line Certificate: The most significant benefits of DW technology are that it protects information, manages digital rights, and conceals secrets, and in the context of e-learning, that involves high-quality images, pdf files etc. DW can also enhance learning efficiency. Furthermore, informationhiding techniques provide opportunities for digital forensic investigators.

on-line certificates in e-learning are still not generally acceptable to educational institutions. Thus, elearning need to develop and create high-quality protected on-line certificates using an efficient DW that can increase trustworthiness in e-learning all over the world. Therefore, this paper has created an on-line DW (inserting VDW and embedding IDW) so that responsible users (usually teachers or administrators) can certificate documents automatically.

As we can see in Fig. 10, the certificate has two kinds of DW, which are VDW and IDW. VDW appears in two shapes, which are the text "De Montfort University" as a rotation from the bottom left corner to the top right corner in Fig. 10. The second shape is the stamp as in the bottom left in Fig. 10. Both shapes can be easily exchanged with the name or stamp of any other institution automatically.

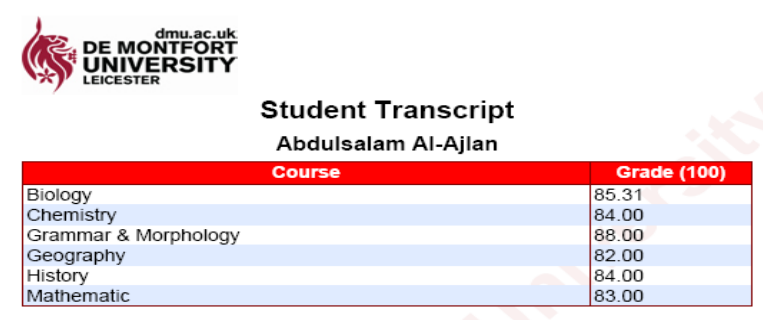

Figure 10: on-line Certificate with DW 
IDW in ECS is hiding a watermark within DMD. The benefits of hiding information are that even if a piece of the image is cropped, the recipient can still obtain the needed message as the watermark is embedded into the message many times. IDW is a cover DMD that cannot be seen by the human eye, but can be discovered by an algorithm.

Extracting a Watermark from DMD by pkunzip Software: Extracting an IDW from DMD by pkunzip software is the second target of our approach. After embedding information into a certificate, we need to extract this information. Thus, we have created a new program that can extract the information that has been embedded into a certificate or into any DMD format. This software is pkunzip but this software depends on other software. The folder "attach_detach" in Fig. 10 has the pkunzip program.

\section{Conclusion and Future Work}

This study contains a survey of e-learning and DW technology, a description of the work needed to be done to satisfy the research problem, and an overview of the results and the contributions we have made. The main challenge of this study has been to find a suitable solution for using DW technology with e-learning systems, and to develop and create a high-quality well-protected online certificate using an efficient DW, which would then promote trust in e-learning certification between institutions all over the world. In addition, the aim has been to develop a DW that enables institutions in different countries to work together, and to share and use certificates without fear of forgery or the dangers of copyright infringement. This paper reduces the levels of forgery and fraud associated with online certification for all institutions across the world.

The most important contribution of this paper has been to improve the use of DW for on-line certificates in e-learning products. Therefore, this paper increases trust between institutions and allows different institutions in different countries to work together and share e-learning certificates. The manner in which this paper has increased trustworthiness to cater for the highly changeable environment of e-learning is through the use of DW and technology its techniques. It has increased trust between institutions in exchanging on-line certificates both easily and cheaply in a flexible manner. In addition, the technology used enables a copyright holder to put in a hidden message within images, moving pictures, sound files, and even raw text. This paper has succeeded in introducing a DW technology that meets the requirements of e-learning by extending the existing architecture of the popular e-learning platform "Moodle".

In the future, when the institutions trust us sufficiently, we will try to build a collaborative e-learning model, gathering all certificates for all institutions all over the world and protecting these certificates by using DW technology. This model will help institutions, teachers and students to have highly secure on-line certificates. We found that there are limitations for using on-line certificates with DW technology in e-learning systems in Saudi Universities, and this is especially the case at Qassim University. Despite the large number of e-learning platforms that exist in institutions in Saudi Arabia, on-line certificates with DW are not used and they continue with normal paper-based certificates. Therefore, in the future, we will try to apply the idea that this paper has introduced to these institutions.

\section{References}

[1] M. Zlomek, Video Watermarking, doctoral diss, Computer Science, Prague University, 2007.

[2] S. Thaker, Software Watermarking Via Assembly Code Transformations, doctoral diss, San Jose State University, 2004.

[3] M. Arnold, et al., Digital Watermarking and Content Protection (2nd edn. Artech House, 2003).

[4] N. Mandhani, Watermarking Using Decimal Sequences Bachelor of Engineering, doctoral diss, Andhra Univ, India, 2004.

[5] R. Chang, and C. Wang, The renovation of the digital watermarking technique, Tamsui Oxford Journal of Mathematical Sciences, 21(2), 2005, 291-304.

[6] Y. Li., C. Tsai, and W. Wu, Reversible fragile watermarking for e-learning media, Proc.9th Pacific Rim Conference on Multimedia, Berlin, 2008, 768--777.

[7] S. Mohanty, and B. Bhargava, Invisible Watermarking Based on Creation and Robust Insertion-Extraction of Image Adaptive Watermarks, Association for Computing Machinery Inc., 5(2), 2008, 1-22.

[8] D. Simitopoulos, et al., Fast Watermarking of MPEG-1/2 Streams Using Compressed-Domain Perceptual Embedding and a Generalized Correlator Detector, EURASIP Journal on Applied Signal Processing, 20(1), 2004, 1088-1106.

[9] I. Cox, and M. Miller, The First 50 Years of Electronic Watermarking, EURASIP Journal on Applied Signal Processing., 2(1), 2002, n126-132,

[10] L. Parameswaran, and K. Anbumani, Content-Based Watermarking for Image Authentication Using Independent Component Analysis, Slovenian Society Informatika, Coimbatore, 3(32), 2008, 299-306.

[11] D. Zheng, Y. Liu, J. Zhao, and A. Saddik A Survey of RST Invariant Image Watermarking Algorithms, ACM Computing Surveys, 39(2), 2007, 1-5.

[12] 14 B. Misra, Digital Watermarking, SE 4C03 Winter, 2005.

[13] A. Al-Ajlan, and H. Zedan, The Extension of Web Services Architecture to Meet the Technical Requirements of Virtual Learning Environments (Moodle), Proc. CCES08-IEEE International Conference on Computer Engineering \& Systems, Egypt, $2008,27-32$.

[14] I. Research, Watermarks: Protecting the image, IBM, 2010.

[15] O. Conlan, Multi-Model: Metadata Driven Approach to Personalised e-Learning, doctoral diss, University of Dublin, 2005.

[16] A. Chavan, and S. Pavri, Open-Source Learning Management with Moodle, linux journal, $2(128), 2004$. 
[17] A. Dong, and H. Li, Ontology-Based Information Integration in Virtual Learning Environment, Proc. 2005 IEEE/WIC/ACM International Conference on Web Intelligence, Washington, USA, 2005, 762-765.

[18] A. Al-Ajlan, and H. Zedan, The Web Services Selection of Virtual Learning Environment Services, Proc. IADIS International Conference WWW/Internet, Vila Real, Portugal, 2007, 50-54.

[19] M. Dougiamas, and P.Taylor, Interpretive Analysis of An Internet-Based Course Constructed Using a New Courseware Tool Called Moodle, Proc. HERDSA conference, Perth, Australia, 2002.

[20] A. Dong, and H. Li, Multimedia Access Platform for Virtual Learning Environment, Proc. of the IEEE International Conference on Electro IT, 2005, 6-14, 22-25.

[21] M. Dougiamas, Moodle, 2012, www.Moodle.org

[22] J. Massy, Study of the e-learning suppliers market in Europe, Master diss, Heriot-Watt University, 2004.

[23] A. Al-Ajlan, and H. Zedan, E-learning (Moodle) Based On Service Oriented Architecture, Proc. EADTU's 20th Anniversary Conference, Portugal, 2007.

[24] A. Al-Ajlan, Service Oriented Computing For Dynamic Virtual Learning Environments, doctoral diss, De Montfort University, UK, 2009.

[25] A. Al-Ajlan, Methodologies, Tools and new Developments For E-Learning, (Ch-10 A Comparative Study between E-learning Features), InTech, Technological Research Institute of São Paulo (IPT), Paulo, Brazil, 2011, 191-214.

[26] A. Al-Ajlan, and H. Zedan, Why moodle, Proc. 12IEEE International Workshop on Future Trends of Distributed Computing Systems (FTDCS), China, IEEE Press, 2008, 58-64.

[27] R. Amir, and A. Zeid, UML-profile for Service-Oriented Architectures, Proc. 19th annual ACM SIGPLAN conference on Objectoriented programming systems, languages, and applications, ACM, USA, 2004, 192-193.

[28] S. Parsa, and M. Damanafshan, Seamless Secure Development of Systems: From Modelling to Enforcement of Access Control Policies, Proc. IEEE/ACS International Conference on Computer Systems and Applications, Amman, $2007,799-806$.

[29] D. Kuhn, V. Hu, W. Polk, and S. Chang, Introduction to Public Key Technology and the Federal PKI Infrastructure, National Institute of Standards and Technology, Government Publication (NIST), U.S, 2001.

[30] M. Huhns, and M. Singh, Service-Oriented Computing: Semantics Processes (John Wiley and Sons Ltd, England, 2005).

[31] F. Chan, R. Bhagwat, and S. Wadhwa, Flexibility Performance: Taguchi's Method Study of Physical System and Operating Control Parameters of FMS, Comput.-Integr. Manuf, 1(23), 2007, 25-37. 